\title{
How can biotechnology improve floriculture?
}

\author{
Fernanda Carlota Nery* (1) \\ Area Editor of the Ornamental Horticulture; Universidade Federal de São João Del Rei (UFSJ), \\ Departamento de Engenharia de Biossistemas, Campus Dom Bosco, São João Del Rei-MG, Brazil
}

Undoubtedly, ornamental plants occupy a prominent place within horticulture due to the relevance of this group of plants to the global economy. In addition to the market around the cultivation and trade of ornamental plants, such plants are the research subject of many scientific groups around the world.

A considerable amount of plant scientists uses biotechnological tools to solve problems or acquire knowledge. Among these, quality products are increasingly sought in the floriculture sector, which has demanded advanced technologies for the high-quality production of seedlings, foliage and flowers to fulfill the market expectations.

Ornamental plants are the group of plants in which the application of biotechnology had a significant expression in the scientific world, with a positive and direct impact on the economy. The boost for that is based on the high added value to the final product.

Biotechnology can also contribute to the preservation of biodiversity, assist in the creation of variability and on the selection of superior genotypes of importance for ornamental plants. Among the strategies included in the biotechnological processes, stands out the development of genetic transformation methodologies associated with tissue culture techniques and the isolation and characterization of the genes of interest. These molecular techniques are important in the manipulation of living collections of genetic resources and the germplasm banks of main ornamental plants and others.

The adoption of technologies regarding biotechnology brings greater global competitiveness to companies that focus on the production and commercialization of ornamental plants, besides adding direct and indirect benefits to producers and the environment.

For many ornamental species, the lack of standardization of products and problems regarding plant health are the main obstacles for Brazilian products exportation. Thus, biotechnology applied to the production of high-quality stock plants, as well as the continuous supply with quality must be a constant concern of flower producers during all the production process stages.

One of the major tasks within breeding programs is the conservation of germplasm, being a reservoir of genes that breeders can access whenever they need to solve specific problems, such as resistance to disease.

The requirement to protect the management of genetic resources to meet national demands must emphasize the conservation, characterization, and availability of germplasm from both native and exotic plants, seeking to guarantee Brazilian food security and expand the international germplasm exchange, in accordance with the national and international policies for the conservation of genetic biodiversity.

Among many biotechnological methods, in vitro banks are one of the most efficient strategies for the in vitro preservation of germplasm collections, which can be done through slow growth techniques and cryopreservation.

Cryopreservation has been seen as a promising technique for the long-term storage of vegetal genetic resources. In addition, it has been considered the technique with the greatest potential to preserve species that propagate vegetatively or that present unviable, intermediate or recalcitrant seeds.

Given the scenario of conservation of genetic diversity and the different responses between different plant species, or even between different tissues of the same species, it becomes increasingly relevant the development of researches regarding in vitro conservation of plants from different species of ornamental plants, through the establishment of micropropagation and cryopreservation protocols.

The acknowledgment of the potential of these biotechnological techniques along with the conservation of genetic resources of flowers and ornamental plants is attractive for both economic and practical reasons, enabling the supply of plant stocks with high phytosanitary standards to producers.

The production of flowers through seeds requires a long cycle of growth and strategies to reduce time through genetic breeding are important to establish transformation methods for the introduction of transgenes, to develop different cultivars with greater attractiveness to the consumer and resistance to diseases. Although they already exist, the genetic transformation protocols for ornamental plants are still incipient, for example, the transformation through Agrobacterium for petunia and rose (ISAAA,

*Corresponding author: fernandacarlota@ufsj.edu.br 
2020). With the CRISPR/Cas9 development, a new world of possibilities has emerged in plant sciences and it has promising applications on floriculture. In the future, the ornamental plants market will be revolutionized by improvements on genome editing techniques (Erpen-Dalla et al., 2019).

The development of new characteristics in ornamental plants is a requirement of the flower market. However, the high heterozygosity and limited genetic information regarding most ornamental plants have restricted breeding programs. Thus, the application of biotechnological techniques such as genetic engineering represents an alternative for obtaining flowers with modified characteristics.

Research on ornamental plants in Brazil and abroad has been pursuing the development of methodologies for the propagation and preservation of several ornamental species in vitro. In addition to such techniques, genetic engineering along with conventional breeding and management techniques has enormous potential to increase agricultural yield, benefit the environment and improve the quality of ornamental plants.

\section{References}

ISAAA. International Service for the Acquisition of Agri-biotech Applications. Available at: $<$ https://www.isaaa.org $>$ Accessed on Nov 30, 2020.

ERPEN-DALLA, C.L.; MAHMOUD, M.L.; MORAES, S.T.; MOU, Z.; GROSSER, W.J., DUTT, M. Development of improved fruit, vegetable, and ornamental crops using the CRISPR/Cas9 genome editing technique. Plants, v.8, n.12, p.601, 2019. https://doi.org/10.3390/plants8120601 